\title{
CONNOTATIONS CULTURELLES DANS LA PERSPECTIVE HUMBOLDTIENNE. ÉTUDE COMPARATIVE DES UNITÉS PHRASÉOLOGIQUES DU FRANÇAIS ET DU POLONAIS
}

\begin{abstract}
A bstract. Dyoniziak Jolanta, Connotations culturelles dans la perspective humboldtienne. Étude comparative des unités phraséologiques du français et du polonais [Cultural connotations in the Humboldtian perspective. Comparative study of the French and Polish phraseological units]. Studia Romanica Posnaniensia, Adam Mickiewicz University Press, Poznań, vol. XXVIII: 2001, pp. 23-33, ISBN 83-232-1144-2, ISSN 0137-2475.
\end{abstract}

The main idea of this article is to indicate the relation of language and culture through reference to Humboldtian philosophy of language and some linguistic theories which take up this problem. The analysis presented here deals with cultural connotations of idiomatic expressions and assuming that languages are representations that render the spirit of nations, it illustrates particular aspects of relationship between language and culture, namely:

- placing expressions in the cultural specificity of particular communities,

- difficulties encountercd while translating them into another language, which are connected with differences in illustrating them (giving their picture).

Examples collected in the further part are to show a certain group of these idiomatic expressions which are motivated immediately by social reality that is specific of either the Polish or the French nation.

1. «Les langues sont des représentations traduisant l'esprit des peuples» (Gaudefroy-Demombynes 1930, p. 84). Ce concept issu du domaine de la philosophie de la langue et dû à Wilhelm von Humboldt constitue notre point de départ dans l'analyse comparative des connotations culturelles de deux langues: française et polonaise. Nous y insistons, car l'analyse en question requiert une approche complexe de nature sociolinguistique, ce qui nous semble bien intéressant.

Humboldt insiste sur une étroite liaison de la langue et de la culture. Sans entrer dans les détails, nous concevons cette dernière, après Humboldt, comme l'action des peuples. Autrement dit, la culture est une activité simultanée de tous les individus d'un même groupe social et la langue est une création qui en jaillit directement. Il en suit que la langue relève de la pratique sociale, dite culture.

1.1. L'intérêt pour la complexité des influences de la culture sur la langue fut d'abord nettement accentué sur le champ de la philosophie. Il faudrait y citer le nom de Johann Herder, auteur des idées qui vont bien dans ce sens. Pour lui la langue 
n'est pas seuleument un simple instrument de communication, mais aussi une source de savoir sur la culture d'une nation qui parle cette langue. Dans la constatation que la langue maternelle constitue une accumulation de savoir d'une nation, conforme à ses propres expériences, ses conditions et à son caractère particulier, Herder insiste nettement sur l'importance de la culture dans la création des comportements linguistiques au sein d'une collectivité. Bref, suivant ses principes on pourrait dire que la langue reflète la vie des nations, car elle en fait partie. L'étude du réel mène aux expériences communes qui s'inscrivent dans la langue en tant que notions par une forme linguistique appropriée (par un mot ou un groupe de mots comme c'est le cas des unités phraséologiques) et sont transmises aux générations suivantes (p.ex. en tant que textes clichés: proverbes, maximes, sentences).

1.2. Pourtant c'est la théorie de Wilhelm von Humboldt qui a influencée beaucoup plus la linguistique que la philosophie de la langue de Herder. Pour éviter de dire que les idées de Humboldt sont une continuation pure de la conception de Herder, il faudrait plutôt constater une autre chose. Notamment, que Humboldt comme d'ailleurs Herder, son contemporain, saisit la langue dans toute sa complexité, c'est-à-dire non seulement en tant que moyen de communication, mais aussi comme un phénomène social. La conception théorique de la langue chez les deux philosophes est analogue, pour ne pas dire identique. Chacune de ces deux conceptions exprime la complexité des phénomènes en question, c'est-à-dire de la langue et de la culture, et souligne qu'il est impossible de les comprendre à part. Il y a une étroite liaison entre la langue et la culture à laquelle un groupe humain a accédé au cours du temps. Chaque collectivité renferme dans la langue son système interprétatif de la réalité extralinguistique basé sur l'expérience propre de la vie sociale. Cette dernière entraine deux activités principales simultanées:

- le processus cognitif qui comprend la perception et la connaissance,

- la communication conçue comme une expression de la connaissance acquise au cours de sa perception et confrontée à tous les phénomènes sociaux liés à cette collectivité.

Dans cette perspective la langue paraît comme un phénomène social complexe, indéniablement lié à l'histoire des civilisations; «Les langues sont soumises à l'action des peuples à qui elles appartiennent, subissent leur influence et sont empreintes de leur caractère particulier» (Gaudefroy-Demombynes 1930, p. 22).

Robert Galisson, un linguiste contemporain, insiste ici sur le concept d'identité collective; «C'est en tant que pratique sociale et produit socio-culturel que la langue est toute pénétrée de culture. Le jeu de symbiose dans lequel fonctionnent langue et culture fait qu'elles sont le reflet réciproque et obligé l'une de l'autre» (Galisson 1991, p. 119).

Le fait que la langue fonctionne à l'intérieur d'un groupe indépendant, dit nation, pousse à ce qu'elle fait partie de sa culture. Sa fonction peut être définie comme la manifestation extérieure de l'esprit du peuple. Quoi qu'il en soit, on admet à l'histoire, à la littérature, à la tradition, c'est-à-dire à la culture le don de créer une certaine 
réalité extralinguistique dans laquelle fonctionne et communique une nation. En tant que facteurs référenciels, ils agissent tous sur la langue et lui donne un lustre.

1.3. Il en résulte la deuxième conception de Humboldt, celle de la langue en tant que force «génératrice»' des nations. Selon Humboldt la langue jouait le rôle d'identificateur collectif déjà à l'époque de la création des communautés primitives. Les Slaves, par exemple, nommèrent leur voisin de l'ouest Niemiec, c'est-à-dire, celui qui ne comprend rien, qui ne parle pas slave. II représentait pour eux une autre culture qui différait premièrement de la leur par la langue. La garantie de l'individualité collective que la langue offre, est liée au fait qu'elle constitue un élément de la pratique sociale. Celle-ci diffère d'une nation à l'autre. Autrement dit, les langues se diversifient d'une manière maniteste. Leur forme est diverse autant qu'est diverse la réalité sociale des nations. N'importe quelle recherche sur ce phénomène démontrerait que la diversité des langues résulte, entre autres, de raisons historiques et sociales. L'influence réciproque des facteurs référenciels comme: histoire, tradition, culture d'une nation donnée est de grande importance dans l'étude des langues, surtout sur le champ d'investigation de l'ethnolinguistique. Les langues connotent de façon différente tout le savoir qui résulte de l'expérience cognitive des communautés linguistiques. On aborde ici la notion de connotation sémantique qui avec la dénotation constituent les moyens linguistiques de l'organisation de la perception et de l'expression de la réalité extralinguistique. «Les connotations sémantiques sont des signes (jugements, émotions) qui sont associées aux designata des objets réels par l'ensemble des sujets parlants, et retenues dans certains faits linguistiques comme métaphores, dérivés, phraséologismes»² (Bartmiński 1999, p. 44). Notre intérêt porte, vu l'objet de cet article, sur les connotations culturelles qui se réfèrent à toute collectivité linguistique, et non seulement aux groupes qui la composent comme, par exemple un milieu social ou un individu (voir p.ex. les connotations stylistiques).

1.4. Les mécanismes connotatifs sont complexes et ils constituent aujourd'hui un champ d'investigation indépendant dans le cadre de la sémantique de la connotation (Kerbrat-Orecchioni, 1977). Appelés valeurs additionnelles par Bloomfield, ou sursignification par Mitterand, ils sont intrinsèques de la langue, ainsi que les mécanismes dénotatifs auxquels ils sont liés. Le décryptage sémantique des unités linguistiques comprend donc une étude à deux niveaux: du contenu dénotatif, c'est-à-dire des informations que véhicule cette unité et qui lui permettent d'entrer en relation avec un objet extralinguistique au cours des processus onomasiologique et sémasiologique; et du contenu connotatif, c'est-à-dire des informations subsidiaires que cette unité véhicule en plus. Dans cette perspective une unité lexicale telle que lion cumule deux sortes de valeurs sémantiques:

\footnotetext{
' Génératrice, c'est-à-dirc celle qui amène à la creation d'identité collcctive.

2 Notre traduction.
} 
- dénotative, qui évoque un animal défini comme un grand mammifère carnivore, un félin à pelage fauve vivant en Afrique et en Asie;

- connotative, qui véhicule l'idée de la puissance et du courage selon les expressions qui suivent:

1) se battre (se défendre) comme un lion - «se battre, se défendre avec fougue, courageusement», pol. bronić się, walczyć jak lew,

2) cour du lion - "grand courage» pol. lwle serce,

3) fam. bouffer (manger) du lion - "être agressif, manifester une certaine volonté de combattre",

4) la part de lion - «la plus grosse part» pol. lwia część,

5) un lion - "personne courageuse, brave»,

6) odwaziny, silny jak lew,

7) lew się w kim budzi - «ktoś staje się odważny»,

8) išć, włazić w paszczę lwa - «iść w najbardziej niebezpleczne miejsce».

La connotation évoquée n'est qu'un rajout sémantique qui relève de la perception collective de la réalité objective propre, entre autres, aux cultures européennes. Ces connotations, appelées symboliques, qui réfèrent aux objets extralinguistiques (chien - fidélité, noir - deuil, etc.) ont la propriété d'être en général partagées par l'ensemble de la communauté. Et parfois, par elle seule, ces associations sont souvent conventionnelles. Ainsi, c'est le blanc qui dans certaines civilisations symbolise le deuil (Kerbrat-Orecchioni 1977, p. 119). L'exemple précité va avec la thèse que chaque langue renferme une vision particulière du monde parce qu'elle recouvre la connaissance commune à une organisation sociale. Tout système représente à sa façon l'univers qui l'entoure, chaque langue ayant une modalité qui lui est propre pour le percevoir et pour le représenter. La thèse en question a été développée dans la linguistique en raison des recherches de Whorf et Sapir. Whorf démontre qu'il n'existent pas deux langues qui se ressemblent jusqu'au point d'exprimer la même réalité sociale. Les réalités dans lesquelles vivent différentes nations sont des réalités à part. C'est principalement dans les mots que se reflète la complexité et l'originalité de chaque vie collective. Robert Galisson évoque le problème posé dans la même optique socio-culturelle. Selon lui, chaque langue dispose d'un appareil de symbolisme référentiel qui vient de la vie sociale et qui permet la consolidation d'une société.

Il en va de même que la langue et la culture sont des phénomènes entre lesquels s'établissent des relations réciproques complexes. La langue ne fonctionne qu'à l'intérieur d'une culture qui constitue pour lui un ensemble des référents extralinguistiques. Ainsi la langue peut-elle être porteur d'identité des nations.

Essayons maintenant d'analyser les faits attestés jusqu'à présent dans le matériel linguistique. Le but du présent article est de trouver les reminiscences de la culture maternelle dans la phraséologie à l'exemple des unités phraséologiques françaises et polonaises. Nous avons choisi d'étudier les connotations culturelles dans les unités phraséologiques, car ces dernières semblent en être une source inépuisable. 
2. Les expressions idiomatiques d'une langue posent beaucoup de problèmes aux apprenants.

2.1. D'abord, parce que d'habitude ces associations de deux ou de plusieurs mots, entièrement figées prennent un sens nouveau, différent du sens de ses constituants. Il en suit qu'il est impossible de les traduire littéralement. Elles se privent du sens propre des élements composants et fonctionnent dans la langue comme un tout sémantique à part, comme dans les exemples qui suivent:

jus de chaussette - "mauvais café», pol. lura,

vif argent - "personne très agitée, très active», pol. żywe srebro,

patte d'oie - «les petites rides divergentes à l'angle externe de l'xil», pol. kurze tapki,

lune de miel - «les premiers temps (littéralement le premier mois) du mariage», pol. miodowy miesiac.

2.2. Ensuite, nombre d'expressions idiomatiques contiennent une valeur sémantique qui peut être considérée globalement comme spécifique d'une langue. Elle ressort ipso facto à ce qu'il est convenu d'appeler la culture et est conséquemment empreignée de sa spécılité.

peigner la girafe - "fiire un travail inutile», équivalent pol. wozić wodę do morza,

merle blanc - "objet introuvable ou d'une extrême rareté», pol. biaty kruk,

une parenté à la mode de Bretagne - "une parenté lointaine entre deux personnes, une relation lointaine entre deux choses», équivalent pol. p.ex. dziesiata woda po kisielu,

tomber de Charybda en Scylla - «échapper à un inconvénient, un danger, etc.; pour tomber dans un autre plus grave», équivalent pol. wpadać z deszczu pod rynnę.

Les exemples ci-dessus font voir que le sens des locutions peut être identique dans deux langues différentes, tandis que les métaphores qui les fondent sémantiquement ne sont pas forcément les mêmes. Autrement dit, le symbolisme référentiel pour le polonais et le français n'est pas toujours analogue.

2.3. Enfin, les locutions figurées sont impossibles à traduire mot à mot parce qu'elles sont chargées d'implicites culturels jusqu'à ce qu'elles n'aient pas d'équivalents dans une autre langue. Elles présentent un système des particularités expressives, liées aux conditions sociales dans lesquelles la langue est actualisée, c'est-àdire à des usages. «Elles sont fixées, traditionnelles et surtout caractéristiques (...) d'un état de la société». (Rey, Chantreau 1993, préface). Il faut admettre que toute expérience collective sert de point d'ancrage à des locutions figurées qui, à leur tour, créditent cette expérience de charges que tout natif mobilise au contact du mot qui se réfère à cette expérience. Autrement dit, nombre d'unités phraséologiques prennent naissance d'une image, d'une métaphore, d'un glissement de sens qui a lieu dans un contexte socio-culturel déterminé. Elles en sont motivées, nous allons le voir dans la partie qui suit. 
Les locutions figurées sont difficiles à comprendre pour les non-natifs d'une langue parce que leur compréhension exige la mise en ouvre d'un certain savoir extralinguistique sur la nation qui parle cette langue. Selon Renata Grzegorczykowa, la difficulté principale dans la communication entre les gens ne vient pas tellement de différences au niveau des systèmes linguistiques, mais surtout des divergences au niveau des expériences et des convictions des sujets parlants (Bartmiński 1999, p. 46). Si on se réfère à la situation de communication entre deux personnes de nations différentes, il est à admettre que les connotations culturelles motivées par un certain ensemble d'expériences collectives, c'est-à-dire par l'histoire, les faits de civilisation, la tradition littéraire, les stéréotypes sociaux, etc., peuvent empêcher la compréhension. Bien que les communautés européennes vivent dans des environnements physiques et sociaux à peu près similaires, elles détiennent à chaque fois une certaine identité culturelle qui s'enferme dans leur langue. Voilà pourquoi les langues ne représentent pas uniquement des différences au niveau phonétique, mais aussi, ce qui est beaucoup plus complexe, au niveau sémantique. Dans différentes collectivités, il existe diverses motivations sémantiques de la perception de la réalité. La personne qui désire apprendre une langue doit s'en rendre compte. Avoir une compétence en langue étrangère, cela signifie connaître des comportements sociaux de la nation qui parle cette langue et participer la culture, bien qu'elle nous soit étrangère. La liaison entre la langue et la culture est indissoluble à tel point qu'on ne peut plus parler de deux phénomènes à part, mais d'un seul.

2.4. Essayons de présenter les idées décrites ci-dessus en forme de schéma, avant de passer à la partie pragmatique.

\section{PRATIQUE DE LA LANGUE}

OBJET (RÉALITE EXTRALINGUISTIQUE)

PERCEPTION: (CATÉGORISATION $\rightarrow$ STRUCTURE CONCEPTUELLE (NOTION))

(chaque langue donne au monde extérieur une forme, le découpe à sa manière)

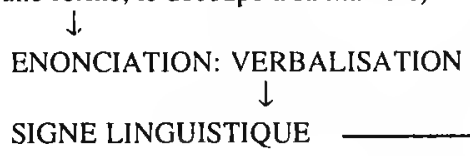

mémoire

(structure conceptuelle)

\section{Albertrandy}

(un fait social, un historien qui avait une bonne mémoire)

\section{mémoire d'Albertrandy}

(expérience collective de la réalité impose la façon de l'organiser et de l'adapter) L'expression devient significative lorsqu'on connaît la connotation qualitative de «bon», qui est une connotation culturelle. 
3. Dans notre présente analyse nous nous appuyons sur les recueils suivants: pour le français - Dictionnaire des expressions et locutions de Rey et Chantereau; pour le polonais - Stownik frazeologiczny języka polskiego de Skorupka et un ouvrage de Krzyżanowski intitulé Madrej gtowie dość dwie stowie. Nous n'avons choisi que certaines expressions idiomatiques parmi un très grand nombre de celles-ci, notamment celles qui se rapportent à une réalité socio-culturelle propre à la nation polonaise et française. Nous les avons mis en groupes selon l'origine de leur valeur connotative.

L'étude des unités phraséologiques que nous trouvons dans les sources citées cidessus montre indéniablement la liaison de la langue et de la culture. Leur origine peut se référer, entre autres, aux:

\section{1. personnages historiques}

coup de Jarnac - "attaque perfide», par allusion à la célèbre botte du chevalier Jarnac qui tua en duel la Châtaigneraie en 1547;

vx. adroit comme un prêtre normand - «très maladroit». L'expression résulte d'un jeu de mots sur l'adjectif gaucher, avec ses connotations péjoratives de maladroit et de saint Gaucher, évêque normand du XI' s.;

polegać jak na Zawiszy - «être sûr de quelqu'un, ne douter de rien». L'expression vient du nom de Zawisza Czarny de Grabowo qui lutta à Grunwald contre les chevaliers Teutoniques, connu dans toute l'Europe comme chevalier sans peur et sans reproche;

pamięć jak u Albertrandego - «une excellente mémoire». Albertrandy Jan (17321808) historien, connu pour sa bonne mémoire;

wyjść jak Zabłocki na mydle - "rater son affaire, subir une perte d'un avantage p.ex. financier». L'expression vient du nom d'un marchand qui transporta par la Vistule, des cargaisons de savon, soit mal chargées, soit cachées au-dessous des cargos pour éviter de payer la douane;

Bismarck w spódnicy - "femme énergique qui exerce l'autorité, qui domine sur d'autres». L'expression vient du nom de Otto von Bismarck, homme d'état prussien (1815 - 1898), qui pratiqua une politique très autoritaire.;

duży jak Łokietek - «dont la hauteur, la taille est inférieure à la moyenne». L'expression vient du nom de roi polonais, Ladislas $I^{\text {er }}$ tokietek de la dynastie des Piast qui était de très petite taille.

3.2. Histoires des contacts internationaux

auberge espagnole - «lieu, et fig., situation, où l'on ne trouve que ce qu'on a soi-même apporté». L'expression vient d'une comparaison avec les auberges d'Espagne, où selon les voyageurs venus du Nord, il était recommandé d'apporter de quoi manger et boire, si l'on ne voulait pas être réduit â la portion congrue;

c'est de l'allemand (du haut allemand) - "c'est incompréhensible». Allusion à l'écriture gothique; plus qu'aux difficultés d'une langue parlée (c'est du chinois, de l'hébreu, et de l'algèbre); 
faire bâtir les châteaux en Espagne - "projets chimériques et irréalisables», l'Espagne fut un pays qui subit de multiples évasions des Maures, grands ravageurs;

gruba Berta - «fenme grosse», du nom du mortier de $422 \mathrm{~mm}$, dont les Allemands se sont servi pendant la Première Guerre mondiale;

cygańskie kupno (targ, praca) - «vol». Les Tziganes, peuple nomade avait une mauvaise renommée en Pologne par le fait qu'ils avaient de l'inclination à voler;

tyle, co Szwedów - «beaucoup». Allusion historique à l'invasion des Suédois, connue sous le nom de Déluge suédois, qui eut lieu en Pologne au XVII' s.;

fort comme un Turc - "doué d'une grande vigueur physique». Le Turc depuis les Croisades symbolisait l'ennemi brutal;

goty jak święty turecki - «très pauvre». Allusion aux derviches, religieux musulmans à moitié nus, qui frappèrent d'étonnement les pèlerins chrétiens se dirigant à Jérusalem;

ivre comme un Polonais - "complètement sô̂l». Référence aux soldats polonais, mercenaires appréciés sous l'Ancien Régime, puis après les guerres de l'Empire.

- Les quatre dernières locutions contiennent une comparaison. Un Polonais, un Turc, święty turecki est un modèle représentant d'une certaine catégorie d'état (ivresse, force physique, pauvreté).

Ivre comme un Polonais. c'est-à-dire très ivre prend toute sa signification lorsqu'on connaît sa connotation quantitative de «très» qui est une connotation culturelle. Cette locution assigne aux Polonais de façon bien stéréotypée le goût excessif pour l'alcool. Les locutions contenant une comparaison ont d'habitude un caractère stéréotypé.

\subsection{Institutions historiques}

Akademia Smorgońska - "école supérieure qui ne garantit aucune formation», connotation qualitative du nom de l'établissement de dressage des ours en Lituanie;

Akademik Smorgoński - «honme grossier»;

face d'abbé - «visage rubicon» (XVI-XVII' s.). La réputation des titulaires d'abbaye, prospères et enviés, était à l'époque classique un intensif par rapport à celles des moines;

table d'abbé - «table où l'on mange bien» (XVI - XVII's.). Le mot abbé, dans la langue classique, évoquait le premier personnage d'une abbaye, et joignait l'idée d'importance, de richesse, à celle de «bon vivant» attachée à moine.

\subsection{Activité littéraire}

accommoder de toutes pièces - «décrier quelqu'un systématiquement, en médire de toutes les façons». L'expression plaisait à Molière; elle est dans «L'Avare», dans "L'École des femmes», dans «George Dandin»;

Ce n'est pas le Pérou - "ce n'est pas une source considérable, une fortune, un gain énorme", Le Pérou dans la mythologie du premier colonialisme espagnol est la terre de l'or; la pauvreté réelle du pays suscite des réemplois géographiques et ironiques; 
Comment peut-on être persan? - la célèbre formule de Montesquieu (Lettres persanes) est souvent rappelée pour simuler un étonnement naîf, souvent fauteur de xénophobie, à propos d'une personne dont l'origine, le comportement, les activités sont étrangères au groupe social;

fier comme Artaban - "très fier». Artabcun est le nom de plusieurs rois parthes de la dynastie des Arsacides, mais ce n'est pas l'histoire du Proche-Orient, ni la personnalité de l'un de ces rois qui a donné naissance à l'expression. Celle-ci est d'origine littéraire, le fier Artaban est un personnage important de l'immense roman historique de Gautier de la Calprenède, intitulé Cléopâtre;

la perfide Albion - "l'Angleterre». La rhétorique politique du XVII' siècle associait volontiers à l'Angleterre l'adjectif perfide (Mme de Sévigné, Bossuet);

fam. laisse bèton - «laisse tomber, abandonne». Formule répandue par une chanson de Renaud (1977);

pleść banialuki - "dire des bêtises, raconter des balivernes». Á l'origine de cette locution est un conte de fées de H. Morsztyn, intitulé Historya ucieszna o zacnej królewnie Banialuce wschodniej krainy;

zawracać komuś gitare - «fatiguer quelqu'un par ses paroles, lui raconter des mensonges». Expression répandue par la chanson de cabaret, intitulée: Wszystkie rybki śpia w jeziorze.

\subsection{Faits de civilisation}

vx. apothicaire sans sucre - «celui qui ne possède rien de ce qui est nécessaire pour exercer sa profession» (fin XVI $I^{e}$.). Jusqu'au règne de Louis XIII, les apothicaires avaient le monopole de la vente du sucre, denrée encore très rare;

bas de laine - "économies". AuXIX" s., les paysans français avaient la réputation de garder leurs économies - notamment en pièces d'or - dans des bas de laine;

fam. aux abonnés absents - renforcement plaisant de absent par allusion à un service - aujourd'hui disparu - des téléphones. S'emploie pour "parti, évanoui, inconscient» (sous l'effet de l'alcool, de la fatigue, etc.), dans rester, être, passer aux abonnés absents;

baiser Lamourette - "réconciliation éphémère». Allusion à une anecdote politique de la Révolution (le député Lamourette avait patronné une réconciliation générale à l'Assemblée législative, en 1792, et les ambassades des adversaires n'avaient eu aucune suite;

les discussions de café du Commerce - «vaines et ridicules en politique», nom assez fréquent de café, brasseries, etc., devenu le symbole du lieu de réunion où la petite bourgeoisie parle de la politique;

échappé de Charenton (variante: bon pour Charenton) - «fou», Charenton est un ancien asile de fous;

mentir comme un arracheur de dents - "effrontément», conme les dentistes qui autrefois, sur les places publiques et dans les foires, offraient leurs services aux volontaires et prétendaient, pour attirer la clientèle que l'opération serait indolore; 
arbuza dostać (dać), dostać (dać) czarna polewke - "en disant d'une femme: refuser un prétendant à la main, repousser ses galanteries». Une habitude d'offrir de la pastèque, ou de la soupe au sang de volaille au prétendant à la main d'une fille à marier vient d'Ukraine et de Podolie. La noblesse la contracta des milieux. populaires. Les parents des filles à marier s'en servirent pour repousser des prétendants importuns;

wpaść jak po ogień - «ne pas rester longtemps en un lieu, avoir de la hâte, être bien pressé». Les motivations historiques de cette expression sont bien intéressantes: il s'agit d'une pratique ancienne des campagnards du temps où les allumettes n'étaient pas encore en usage et le matin quand le feu se fut éteint, il fallait aller le chercher chez les voisins;

fam. oddać kogo do czubków (uciekt do czubków) - «se dit des personnes atteintes de troubles mentaux, aliénées, folles». Référence à l'ordre appelé en Pologne Bonifracia, qui au XVI s. s'occupèrent du traitement des fous;

drzeć ze soba koty - "se chamailler, avoir maille à partir avec qqn». Selon Krzyzanowski a l'origine de cette expression était un jeu athlétique appelé Kocury, qui consistait à faire lâcher à son adversaire un grand cube de bois;

psu na budę - "pour un résultat nul, pour rien, cela ne vaut rien". Une belle métaphore issue de la cour de ferme où les paysans «bâclaient» la niche de planches déjà pourries ou de quelques ramures avec un peu de paille. Une construction si provisoire n'était pas toujours pour le chien un abri suffisant contre la pluie.

3.6. Même si les expressions idiomatiques de deux langues différentes possèdent la même origine culturelle, par exemple, la Bible, il convient de noter que la spécialisation sémantique postérieure de ces unités peut procéder de façon indépendante dans deux systèmes linguistiques et amener, soit à l'oubli de la motivation d'origine, soit à un déplacement sensible envers la référence étymologique. La motivation sémantique des unités phraséologiques peut varier en fonction d'usage dans le contexte socio-culturel:

p.ex. aller chez Abraham - «mourir», locution issue de la Bible qui garde toujours la motivation biblique;

pol. iść na kwaśne piwko do Abramka - «mourir», expression motivée par la réalité polonaise $d u X V I^{e}$ s. Le prénom Abraham, autrefois très souvent rencontré dans les milieux chrétiens, ne fonctionne plus que chez les Juifs, propriétaires des auberges, où ils vendaient de la bière de faible qualité.

Comme nous l'avons pu voir, les unités phraséologiques sont profondément enracinées dans la tradition socio-culturelle des nations. Elles sont chargées d'implicites culturels que nous avons appelés connotations culturelles. Elles démontrent à quel point les réalités sociales ne sont pas équivalentes. La langue est un fait social, elle tire sa substance des domaines d'expérience sociale qui constitue le dénominateur commun des connaissances indispensables pour que s'instaure l'échange entre individus. 


\section{BIBLIOGRAPHIE}

Barımiński J. (1999), Językowy obraz swiata, WUMCS, Lublin.

Bytniewski P. (1991), Język i kultura w koncepcji E. Sapira i B.L. Whorfa, (in:) Język a kultura (sous réd.) Puzynina J., Bartmiński J., vol. 2, Wiedza o kulturze, Wrocław 1991.

Galisson R. (1991), De la langue à la culture par les mots, Clé international, Paris.

Gaudefroy-Demombynes J. (1930), L'CEuve linguistique de Humboldt, Paris.

Guiraud P. (1972), La sémantique. PUF, Paris.

Guiraud P. (1973), Les locutions françaises, PUF, Paris.

Kerbral-Orecchioni K. (1977), La connotation, Presses Universitaires de Lyon, Lyon.

Kochan B., Zaręba L. (1999), Idiomy polsko-francuskie, PWN, Warszawa.

Krzyżanow ski J. (1967), Madrej glowie dość dwie stowie, PIW, Warszawa.

Rastier F. (1987), Sémantique interprétative, PUF, Paris.

Rey A., Chantreau S. (1993), Dictionnaire des expressions et locutions, le Robert, Paris.

Sch aff A. (1964), Jezzk a poznanie, PIW, Warszawa.

Sk orupka S. (1967), Stownik frazeologiczny języka polskiego, WP, Warszawa.

Sy pnicki J. (1998), O roli elementów kulturowych w leksyce, (in:) Język a kultura. TPPW, Wroclaw.

Whorf L. B. (1982), Język, myśl, rzeczywisłość, PIW.

Zarçba L. (1995), Polsko-francuski słownik frazeologiczny, PWN, Warszawa. 